\title{
A Fast Full Search Scheme for Rate-Distortion Optimization of Variable Block Size and Multi-frame Motion Estimation
}

\author{
Yan-Ho Kam and Wan-Chi Siu \\ Centre for Multimedia Signal Processing \\ Department of Electronic and Information Engineering \\ The Hong Kong Polytechnic University \\ Hung Hom, Kowloon, Hong Kong \\ Email: enwcsiu@polyu.edu.hk
}

\begin{abstract}
Two new fast full search (FFS) methods are proposed which can improve the performance of the ratedistortion optimized video encoding process. The first method combines the concepts of both conventional sum of absolute difference (SAD) reuse method and row-based partial distortion search (PDS) together to make the performance largely better than these two conventional methods for variable block size motion estimation. The second method speeds up the multi-frame motion estimation process by using the previous results obtained in considered reference frames to set extra thresholds for rejecting search points sooner in reference frames. Furthermore, these two proposed methods can be easily combined to form a very effective motion compensation scheme. On average, a $62 \%$ and $52 \%$ reduction in terms of computational complexity of integer variable block size and multi-frame motion estimation can be achieved without any video quality degradation as compared with that of the conventional PDS and conventional SAD reuse method respectively.
\end{abstract}

\section{INTRODUCTION}

The block-based motion estimation (ME) technique has been widely used in many modern video-coding standards such as the H.264 [1]. It is used to reduce the redundancy between successive frames in a video. In this video-coding scheme, a frame is divided into a number of non-overlapping macroblocks (MBs). By applying $\mathrm{ME}$ in a video encoder, the best match of each $\mathrm{MB}$ in the current frame is searched from a reference frame, and then the spatial displacement between the current MB and the best match is defined as a motion vector (MV). Best match means that the distortion measure between the MB in the current frame and the match in the reference frame is the minimum. One of the most frequently used distortion measure is the sum of absolute differences (SAD). MVs and residue errors are then coded into bitstreams, and are transmitted to a decoder or stored.
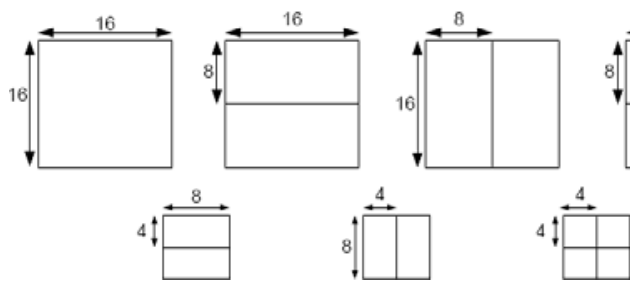

Fig. 1. 7 different block sizes adopted by H.264 standard
The H.264/AVC standard has adopted variable block sizes (VBS) [2] and multiple reference frames (MRF) [3] for ME to improve the quality of encoded video sequences at the expenses of high computational complexity.

As for VBS, H.264 supports seven different block sizes with a tree-structured hierarchical MB partitions as shown in Fig. 1 instead of fixed 16x16 block size adopted in many prior standards such as MPEG-2, H.263, etc. The original approach of VBS ME is to perform ME for all blocks and all block sizes, and then select the best blocks size that the distortion is minimized.

To encode a $\mathrm{P}$ frame, usually, one reference frame is used. However, the best match may exist in another decoded frame so that MRF-ME was proposed to allow using more than one reference frames for motion searches.

In order to reduce the computational effort for ME, some fast methods such as edge oriented block matching algorithm (EOBMA) [4], edge-assisted search algorithm (EAS) [5], partial distortion search (PDS) [6], SAD reuse algorithm [7], successive elimination algorithm (SEA) [8], and adaptive PDS using clustered pixel matching error characteristic (CPME-PDS) [9] were proposed.

In PDS, a candidate block can be rejected before the exact SAD value between a current block and the candidate block is fully computed once the partial SAD (PSAD) value is larger than or equal to the already found minimum SAD cost from previously checked candidate positions. By using this method, many unnecessary computations can be saved. For row-based PDS, PSAD value is updated after each time the SAD value for one row is computed, and then the PSAD value will be checked to judge whether the current candidate can be rejected.

SAD reuse algorithm is a lossless fast algorithm for VBS-ME. Initially, a full search (FS) algorithm is performed for all $4 \times 4$ blocks within each MB inside their search ranges so that for each $4 \times 4$ block, a set of SAD values between the block and all candidates is obtained. Since any larger block is just a combination of two smaller blocks, the SAD value for each $8 \times 4$ or $4 \times 8$ block can be constructed from the SAD values for two $4 \times 4$ blocks by a simple addition. Similarly, the SAD value between any block and each of its candidates can be obtained by adding the SAD values for two corresponding smaller blocks together. Since motion searches are performed for $4 \times 4$ block size only, the number of computations is largely reduced.

This paper proposes a fast full search scheme for speeding up the VBS and MRF-ME processes without quality degradation. The scheme includes two new ideas, namely partial SAD reuse PDS 
(PSADR-PDS) and extra threshold MRF-PDS (ETMRF-PDS) respectively. The proposed scheme is presented in Section 2. Experimental results are shown and discussed in Section 3. Section 4 gives a conclusion of the investigation.

\section{Proposed AlgorithM}

The proposed algorithm is composed of two coding methods, PSADR-PDS and ETMRF-PDS.

\section{A. Partial SAD Reuse PDS (PSADR-PDS)}

This is a lossless fast method for VBS-ME which combines the advantages of conventional SAD reuse algorithm and row-based PDS with regular scanning patterns together.

For each MB, there are 16 non-overlapping $4 \mathrm{x} 4$ regions inside. In this proposed method, for each $4 \times 4$ region, there is a buffer to store the PSAD value obtained by row-based PDS for each candidate search point within the search window in the reference frame. Instead of the traditional top-down scanning pattern, the row-based PDS makes use of regular scanning patterns as shown in Fig. 2.

By investigating the average spatial distribution of pixel matching errors [9], it is found that errors with similar magnitude tend to appear together within a region. It is because natural images are dominated by low-frequency components. Due to this clustering effect, row-based PDS using traditional top-down scanning pattern cannot functions well. If one row of pixels is considered during block matching and the error is found to be small, the error for the row of pixels below is likely to be small too. As a result, more rows of pixels are required to be considered for accumulating the PSAD value until it is beyond the already found minimum SAD. But if the regular patterns are used, after considering one row of pixels, the matching process will jump to a farther row so that the probability of reaching a large error is larger.

Since row-based PDS is used, some search points may be rejected before the SAD values for all rows are computed, and only PSAD values are obtained. Then if the target block is partitioned into non-overlapping $4 \times 4$ regions, PSAD values for different $4 \times 4$ regions can be obtained. These PSAD values are then stored in appropriate buffers, and will be used by smaller block sizes. Information about which rows are already considered for each search point is also stored.
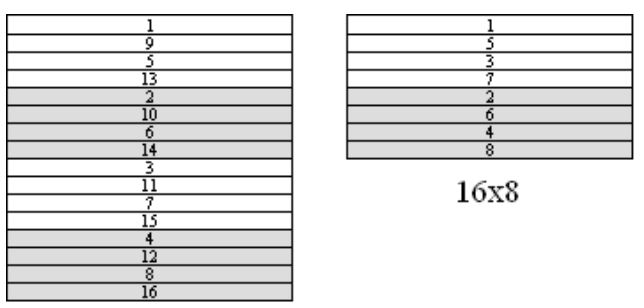

$16 \mathrm{x} 8$ $16 \times 16$

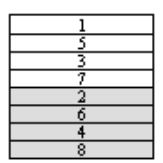

$8 \times 8$

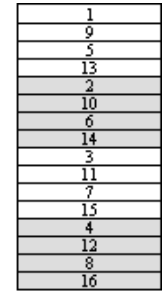

$8 \times 16$

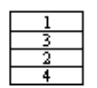

$4 \mathrm{x} 4$

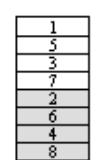

$4 \mathrm{x} 8$

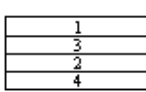

$8 \mathrm{x} 4$
Fig. 2. Regular scanning patterns for row-based PDS
The order of block size to be considered in this algorithm is:

$16 \times 16 \rightarrow 16 \times 8 \rightarrow 8 \times 16 \rightarrow 8 \times 8 \rightarrow 8 \times 4 \rightarrow 4 \times 8 \rightarrow 4 \times 4$

which is the reverse of the order in the conventional SAD reuse algorithm.

For example in Fig. 3, assuming that a candidate search point for a 16x16 block is rejected after computing the PSAD value from 5 rows $\left(1^{\text {st }}, 3^{\text {rd }}, 5^{\text {th }}, 9^{\text {th }}\right.$ and $13^{\text {th }}$ rows $), 16$ PSAD values for 16 different $4 \times 4$ regions are obtained and stored in buffers. Since this $16 \times 16$ block is composed of two 16x8 blocks, the PSAD values corresponding to the upper part of the $16 \times 16$ block can be reused in the PDS for the upper 16x8 block. Therefore, when the PDS is performed for the upper $16 \times 8$ block, at the same candidate search point, an intermediate PSAD value can be obtained simply by adding the upper 8 buffer values together as shown in Fig. 4 since the SAD values for the $1^{\text {st }}, 3^{\text {rd }}$ and $5^{\text {th }}$ rows were already computed. In this case, the computation for the SAD values for 3 rows is saved. After performing ME for two $16 \times 8$ blocks, the updated PSAD values for all $4 \times 4$ regions within the MB are obtained so the values stored in all buffers can be updated. Similarly, the new buffer values can be used to compute the intermediate PSAD values for $8 \times 16$ blocks.

The example shown in Fig. 3 and 4 also shows that considering block sizes in the order of: $16 \times 16 \rightarrow 16 \times 8 \rightarrow 8 \times 16 \rightarrow 8 \times 8 \rightarrow 8 \times 4$ $\rightarrow 4 \times 8 \rightarrow 4 \times 4$ makes the block matching process after reusing previously determined results smooth. It is because the SAD values to be reused always correspond to complete rows of pixels so that we can continue to consider the remaining rows after reusing previous results. Reversely, if the order of: $4 \times 4 \rightarrow 4 \times 8 \rightarrow 8 \times 4 \rightarrow$ $8 \mathrm{x} 8 \rightarrow 8 \mathrm{x} 16 \rightarrow 16 \mathrm{x} 8 \rightarrow 16 \mathrm{x} 16$ is used, the $\mathrm{SAD}$ values to be reused may correspond to unconnected strips of pixels like the example shown in Fig. 5. In Fig. 5, the SAD values for pixels with white color are computed before and reusable. In this case, it is inconvenient to consider the remaining pixels since they are separated.

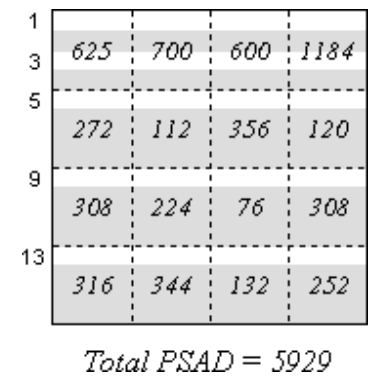

Fig. 3. 16 PSAD values for 16 different $4 \times 4$ regions are obtained after performing PDS in a candidate point

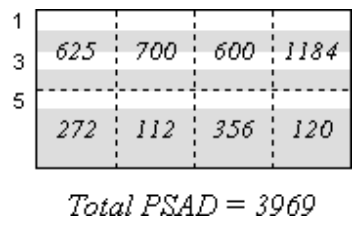

Fig. 4. The intermediate PSAD value for the upper $16 \times 8$ block can be obtained by adding 8 buffer values together 


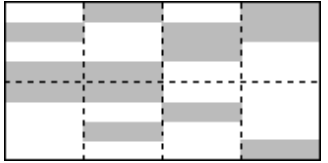

Fig. 5. SAD values to be reused may correspond to unconnected strips of pixels if block sizes are considered in a bottom-up order. Note that the SAD values for pixels with white color are computed before and reusable

On the other hand, by using the regular patterns shown in Fig. 2, the row of pixels will be considered every other three rows for each vertical scan. Because of this arrangement, when the matching is done for a target block, the PSAD results for all $4 \times 4$ regions involved in the target block will be updated after each time four rows are considered. This is good since it makes the progresses of updating the PSAD values for different $4 \times 4$ regions roughly evenly, and hence the PSAD reusing scheme can work more effective.

\section{B. Extra Threshold Multiple Reference Frame PDS (ETMRF-PDS)}

The conventional approach of performing MRF-ME is to perform search and look for the best match in each reference frame independently, and then select the best reference frame and its corresponding motion vector that requires the smallest cost. This proposed method is a lossless fast method that makes use of previous results for considered reference frames to speed up the search in remaining reference frames.

\section{1) For inter $16 \times 16,16 \times 8,8 \times 16$ and $8 \times 8$ modes}

If rate-distortion optimization (RDO) $[10,11]$ is applied, the best match found in each reference frame should minimize the motion cost,

$$
\mathrm{J}_{\text {motion }}(\mathrm{m} \mid \mathrm{r})=\operatorname{SAD}(\mathrm{m} \mid \mathrm{r})+\lambda\left(\mathrm{MV}_{-} \operatorname{cost}\left(\mathrm{m}_{\mathrm{p}}, \mathrm{m}\right)\right),
$$

where $\mathrm{m}=\left(\mathrm{m}_{\mathrm{x}}, \mathrm{m}_{\mathrm{y}}\right)$ is the candidate $\mathrm{MV}, \mathrm{r}$ is the reference frame index, $\operatorname{SAD}(\mathrm{m} \mid \mathrm{r})$ is the $\mathrm{SAD}$ value between the current block and the candidate block pointed by MV $\mathrm{m}$ in reference frame $\mathrm{r}, \lambda$ is the Lagrangian multiplier, $\mathrm{m}_{\mathrm{p}}$ is the motion predictor, and MV_cost $\left(\mathrm{m}_{\mathrm{p}}\right.$, $\mathrm{m}$ ) is the MV difference cost which is the number of bits required for encoding the difference between $\mathrm{m}$ and $\mathrm{m}_{\mathrm{p}}$. Then, the best reference frame is selected by minimizing

$$
J_{\text {ref }}(r)=S_{\text {best }}(r)+\lambda\left(M V_{-} \text {cost } t_{\text {best }}(r)+R E F \_\operatorname{cost}(r)\right) \text {, }
$$

where $\mathrm{SAD}_{\text {best }}(\mathrm{r})$ is the $\mathrm{SAD}$ value for the best match found in reference frame $r, M V$ cost $_{\text {best }}(r)$ is the MV difference cost for the best match found in reference frame $r$, and REF_cost $(r)$ is the reference frame index cost which is the number of bits required for encoding the reference frame index $r$.

Therefore, the MRF-ME process is equivalent to finding a match from all reference frames that minimizes

$$
\mathrm{J}(\mathrm{m}, \mathrm{r})=\mathrm{J}_{\text {motion }}(\mathrm{m}, \mathrm{r})+\lambda\left(\mathrm{REF}_{-} \operatorname{cost}(\mathrm{r})\right) \text {. }
$$

From equation (3), it implies that the MRF-ME process is not necessary to be done by performing MEs in all reference frames separately, but can be completed by searching all candidate points in all reference frames at a time.

There is no advantage in finding the best match from all reference frames at a time if exhaustive search is used because the motion cost for all candidate matches is still needed to be computed, and the number of operations used to compute the SAD values are the same as the original MRF-ME method. Moreover, it is beneficial if the PDS is used instead of the exhaustive search. We want to find out one candidate point with a cost which is the least among all candidates in all reference frames. After searching in one or more reference frames, candidates in the remaining reference frames which have not yet been considered should be rejected if their cost is larger than the minimum cost, $\mathrm{J}_{\min }$, found in considered reference frames. Therefore, if the PDS is applied to all reference frames at a time, candidates in reference frame $\mathrm{r}$ can be rejected when

$$
\mathrm{PJ}_{\text {motion }}(\mathrm{m}, \mathrm{r})+\lambda\left(\mathrm{REF}_{-} \operatorname{cost}(\mathrm{r})\right) \geq \mathrm{J}_{\text {min }},
$$

where $\mathrm{PJ}_{\text {motion }}(\mathrm{m}, \mathrm{r})$ is the accumulated partial motion cost for a candidate pointed by MV $\mathrm{m}$ in reference frame $\mathrm{r}$, and

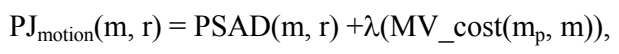

where $\operatorname{PSAD}(m, r)$ is the accumulated partial $S A D$ value.

In other words, the minimum cost, $\mathrm{J}_{\min }$, found in considered reference frames can be used as an extra threshold $\mathrm{TH}$ for the rejection during the PDS in reference frames which have not yet been considered.

\section{2) For inter $8 \times 4,4 \times 8$ and $4 \times 4$ modes}

Regarding the H.264 standard, for inter $8 \times 4,4 \times 8$ and $4 \times 4$ modes, all blocks in each $8 \times 8$ region must be predicted from the same reference frame. Therefore, we need to select a reference frame for each $8 \times 8$ region instead of select a reference frame for each block. If RDO is applied, after finding the best match for each block in each reference frame, the best reference frame is selected by minimizing

$$
J_{r e f}(r)=\left\{\begin{array}{lr}
\lambda\left(\mathrm{REF}_{-} \operatorname{cost}(\mathrm{r})\right)+\sum_{\text {block } k 0}^{1} J_{\text {best }}(\text { block }, r) & \text { for inter } 8 \mathrm{x} 4 \& 4 \mathrm{x} 8 \text { modes } \\
\lambda\left(\mathrm{REF} \_\operatorname{cost}(\mathrm{r})\right)+\sum_{\text {block }=0}^{3} J_{\text {best }}(\text { block }, r) & \text { for inter } 4 \mathrm{x} 4 \text { mode }
\end{array}\right.
$$

where $J_{\text {best }}($ block, $r)$ is the motion cost for the best match found in reference frame $r$ for the block indexed by 'block'.

Instead of applying PDS in each reference frame independently, as before, results from the previously considered reference frames can be used to speed up the MRF-ME process. After the search has been done in $\mathrm{N}$ reference frames for all blocks in a target $8 \times 8$ region, a minimum cost,

$$
J_{\min }=\min _{r=1,2, \ldots, x}\left(J_{r e f}(r)\right),
$$

among all considered reference frames is obtained. Then, for applying PDS in remaining reference frame $r$ for each block, candidates should be rejected when

$$
P J_{\text {motion }}(b+1, m, r)+\lambda\left(R E F_{-} \text {cost }(r)\right)+\sum_{\text {block }=1}^{b}\left(J_{\text {best }}(\text { block }, r)\right) \geq J_{\text {min }},
$$

where $b$ is the number of blocks that the best matches for them in reference frame $\mathrm{r}$ are found before, and $\mathrm{PJ}_{\text {motion }}(\mathrm{b}+1, \mathrm{~m}, \mathrm{r})$ is the accumulated partial motion cost for a candidate pointed by motion vector $\mathrm{m}$ in reference frame $\mathrm{r}$ for a target block indexed by $\mathrm{b}+1$.

If all candidates in reference frame $r$ for a target block are rejected by using the extra threshold $T H=J_{\min }$, we can even stop searching for the best matches of all blocks which have not yet been considered in this reference frame as the final cost $J_{r e f}(r)$ must be larger than or equal to $J_{\text {min }}$. 


\section{EXPERIMENTAL RESULTS}

In our experimental work, 5 QCIF and 5 CIF video sequences with motion activities varying from small to large are encoded by using the H.264/AVC verification model JM9.0 [7] embedded with the proposed methods. All test sequences contains 300 frames. The search range was set to \pm 16 , and the quantization parameter was set to 30 . Besides, intra prediction modes, 7 variable block sizes as well as 5 reference frames were allowed. RDO and median predictors were applied. All sequences were encoded in IPPP... format.

Table 1 shows the comparison of the average number of operations (additions + subtractions + compares + absolute operations) required for integer $\mathrm{ME}$ for each $\mathrm{MB}$ among the traditional PDS, SAD reuse algorithm, PSADR-PDS, ETMRF-PDS and the proposed PSADR-PDS + ETMRF-PDS scheme.

From the results, it is found that the proposed PSADR-PDS method can, on average, reduce $58 \%$ and $47 \%$ of the computation as compared with that of the traditional PDS and SAD reuse algorithm respectively. Regarding the proposed ETMRF-PDS method, it is compared with the traditional PDS algorithm, and the average saving is $17 \%$. If the combined version of the PSADR-PDS and ETMRF-PDS is used, the performance can further be improved. It reduces the average number of operations by $55-74 \%$ with respect to the traditional PDS algorithm and $41-59 \%$ with respect to the SAD reuse algorithm. The average reductions are $62 \%$ and $52 \%$ as compared with two traditional algorithms.

\section{CONCLUSION}

In this paper, two new lossless fast ideas have been proposed to improve the performance of the rate-distortion optimized video encoding process. PSADR-PDS combines the SAD reuse algorithm and row-based PDS together to speed up the block matching processes for variable block size ME by reusing partial SAD results. DTMRF-PDS speeds up the multi-frame ME process by using results previously searched from some reference frames to set extra thresholds to reject search points in other reference frames in advance. In addition, a very effective ME scheme is resulted by combining the two proposed ideas together. This scheme is substantially better than two representative fast full algorithms for variable block size and multi-frame ME process. Experimental results show that compared with the traditional PDS and SAD reuse algorithm respectively, a $62 \%$ and $52 \%$ saving in terms of the number of operations can be achieved by using the combined scheme, on average.

Acknowledgement: This work is supported by the Centre for Multimedia Signal Processing, Department of Electronic and Information Engineering, Hong Kong Polytechnic University and the Research Grant Council of the Hong Kong SAR Government (PolyU 5234/03E).

\section{REFERENCES}

[1] Joint Video Team of ITU-T and ISO/IEC JTC 1, "Draft ITU-T recommendation and final draft international standard of joint video specification (ITU-T Rec. H.264 | ISO/IEC 14496-10 AVC)," Joint Video Team (JVT) of ISO/IEC MPEG and ITU-T VCEG, Document JVT-G050, December 2003.

[2] M. H. Chan, Y. B. Yu, and A. G. Constantinides, "Variable size block matching motion compensation with applications to video coding," IEE Proc. on Communications, Speech and Vision, vol. 137, pt. 1, no. 4, pp. 205-212, August 1990.

[3] T. Wiegand, X. Zhang, and B. Girod, "Block-based hybrid video coding using motion-compensated long-term memory prediction," Proc. of Picture Coding Sym., Berlin, Germany, pp. 153-158, September 1997.

[4] Y. L. Chan and W. C. Siu, "Edge oriented block motion estimation for video coding," IEE Proc. on Vision, Image and Signal Processing, vol. 144, no. 3, pp. 136-144, June 1997.

[5] Y. L. Chan and W. C. Siu, "An efficient search strategy for block motion estimation using image features," IEEE Trans. on Image Processing, vol. 10, no. 8, pp. 1223-1238, August 2001.

[6] S. Eckart and C. Fogg, "ISO/IEC MPEG-2 software video codec," Proc. SPIE, vol. 2419, pp. 100-118, 1995 .

[7] H.264/AVC reference software JM9.0, http://iphome.hhi.de/suehring/tml/.

[8] W. Li and E. Salari, "Successive elimination algorithm for motion estimation," IEEE Trans. on Image Processing, vol. 4, no. 1, pp. 105107, January 1995.

[9] K. C. Hui, W. C. Siu, and Y. L. Chan, "New adaptive partial distortion search using clustered pixel matching error characteristic," IEEE Trans. on Image Processing, vol. 14, no. 5, pp. 597-607, May 2005.

[10] A. Ortega and K. Ramchandran, "Rate-distortion methods for image and video compression: An Overview," IEEE Signal Processing Mag., vol. 15, pp. 23-50, November 1998.

[11] G. J. Sullivan and R. L. Baker, "Rate-distortion optimization for video compression,” IEEE Signal Processing Mag., vol.15, pp.74-90, November 1998 .

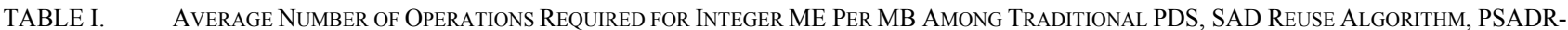
PDS, ETMRF-PDS AND THE PROPOSED PSADR-PDS + ETMRF-PDS SCHEME

\begin{tabular}{|c|c|c|c|c|c|c|c|c|c|c|c|}
\hline \multirow[b]{3}{*}{ Sequence } & \multirow[b]{3}{*}{ Format } & \multicolumn{10}{|c|}{ Method } \\
\hline & & \multirow{2}{*}{$\begin{array}{l}\text { Trad. PDS } \\
\text { Avg. no. of } \\
\text { operations }\end{array}$} & \multirow{2}{*}{$\begin{array}{l}\text { SAD reuse } \\
\begin{array}{l}\text { Avg. no. of } \\
\text { operations }\end{array}\end{array}$} & \multicolumn{3}{|c|}{ PSADR-PDS } & \multicolumn{2}{|c|}{ ETMRF-PDS } & \multicolumn{3}{|c|}{ PSADR-PDS + ETMRF-PDS } \\
\hline & & & & $\begin{array}{l}\text { Avg. no. of } \\
\text { operations }\end{array}$ & $\begin{array}{c}\text { Saving } \\
\text { w.r.t. } \\
\text { trad. } \\
\text { PDS }\end{array}$ & $\begin{array}{c}\text { Saving } \\
\text { w.r.t. } \\
\text { SAD } \\
\text { reuse }\end{array}$ & $\begin{array}{l}\text { Avg. no. of } \\
\text { operations }\end{array}$ & $\begin{array}{c}\text { Saving } \\
\text { w.r.t. } \\
\text { trad. } \\
\text { PDS }\end{array}$ & $\begin{array}{l}\text { Avg. no. of } \\
\text { operations }\end{array}$ & $\begin{array}{c}\text { Saving } \\
\text { w.r.t. } \\
\text { trad. } \\
\text { PDS }\end{array}$ & $\begin{array}{c}\text { Saving } \\
\text { w.r.t. } \\
\text { SAD } \\
\text { reuse }\end{array}$ \\
\hline Mother daughter & QCIF & 4305187 & 4480544 & 1975037 & $54 \%$ & $56 \%$ & 3639433 & $15 \%$ & 1819862 & $58 \%$ & $59 \%$ \\
\hline News & QCIF & 4502579 & 4476690 & 2024826 & $55 \%$ & $55 \%$ & 3800357 & $16 \%$ & 1863892 & $59 \%$ & $58 \%$ \\
\hline Silent & QCIF & 5236407 & 4442606 & 2163204 & $59 \%$ & $51 \%$ & 4326402 & $17 \%$ & 1976511 & $62 \%$ & $56 \%$ \\
\hline Coastguard & QCIF & 8739421 & 4466845 & 2824037 & $68 \%$ & $37 \%$ & 6807110 & $22 \%$ & 2477432 & $72 \%$ & $45 \%$ \\
\hline Container & QCIF & 5204612 & 4490160 & 2319181 & $55 \%$ & $48 \%$ & 4595384 & $12 \%$ & 2198409 & $58 \%$ & $51 \%$ \\
\hline Mother daughter & CIF & 4423706 & 4534661 & 2204726 & $50 \%$ & $51 \%$ & 3613662 & $18 \%$ & 1996958 & $55 \%$ & $56 \%$ \\
\hline News & CIF & 4334177 & 4522683 & 2156089 & $50 \%$ & $52 \%$ & 3542407 & $18 \%$ & 1967380 & $55 \%$ & $56 \%$ \\
\hline Silent & $\mathrm{CIF}$ & 5856254 & 4467194 & 2320317 & $60 \%$ & $48 \%$ & 4786019 & $18 \%$ & 2110906 & $64 \%$ & $53 \%$ \\
\hline Coastguard & CIF & 10225873 & 4474784 & 3082027 & $70 \%$ & $31 \%$ & 7607042 & $26 \%$ & 2620074 & $74 \%$ & $41 \%$ \\
\hline Container & CIF & 6590607 & 4537525 & 2767704 & $58 \%$ & $39 \%$ & 5825324 & $12 \%$ & 2611897 & $60 \%$ & $42 \%$ \\
\hline & & & & Average: & $58 \%$ & $47 \%$ & Average: & $17 \%$ & Average: & $62 \%$ & $52 \%$ \\
\hline
\end{tabular}

